\title{
Regionalization in thoracic surgery: The importance of the team
}

Gail E. Darling, MD, FRCSC

Feature Editor's Note-Regionalization of health care remains a controversial topic and has been proposed as a way to improve outcomes in patients undergoing complex thoracic surgical procedures based on studies correlating hospital and surgeon volume to outcomes. In this month's Journal, we have 2 Invited Expert Opinion articles addressing the topic of regionalization. The first article, by Dr Gail Darling, who is a well-recognized expert in this area, is entitled "Regionalization in Thoracic Surgery: The Importance of the Team" and describes the benefits and challenges of implementing regionalization in Ontario, Canada. The author points out that the benefits of regionalization are not just about the volume of surgery but the availability of team expertise providing a critical mass of specialty providers and allowing optimization of patient outcomes. While a single-payer system in Canada has facilitated the regionalization of thoracic surgical care in Ontario, there are examples of large health systems in the United States that have regionalized complex surgical procedures to high-volume hospitals within their health care networks.

The second article is written by a leading thoracic surgery health services research group led by Dr Varun Puri on the "Economic Implications of Regionalization in the United States." The authors provide an excellent overview of the potential benefits and consequences of volumebased regionalization of thoracic surgery care. They also describe a decision analytic model their group has developed that could be used to evaluate the cost-effectiveness of regionalization in the United States. Regionalization of care remains a complex and controversial topic of interest to thoracic surgeons. Important lessons can be learned from the regionalization of thoracic surgery in Ontario, but the benefits must be carefully considered with the

\footnotetext{
From the Division of Thoracic Surgery, Department of Surgery, University of Toronto; Clinical Lead Thoracic Cancers and High Risk Lung Cancer Screening, Ontario Health, Cancer Care Ontario; and Thoracic Cancers and High Risk Lung Cancer Screening and Toronto General Research Institute, Toronto General Hospital, Toronto, Ontario, Canada.

Received for publication Jan 29, 2020; revisions received May 27, 2020; accepted for publication June 7, 2020; available ahead of print July 18, 2020.

Address for reprints: Gail E. Darling, MD, FRCSC, Toronto General Hospital, 200 Elizabeth St, 9N-955, Toronto, Ontario, Canada M5G 2C4 (E-mail: gail. darling@uhn.ca).

J Thorac Cardiovasc Surg 2021;161:323-9

$0022-5223 / \$ 36.00$

Copyright (C) 2020 by The American Association for Thoracic Surgery

https://doi.org/10.1016/j.jtcvs.2020.06.138
}

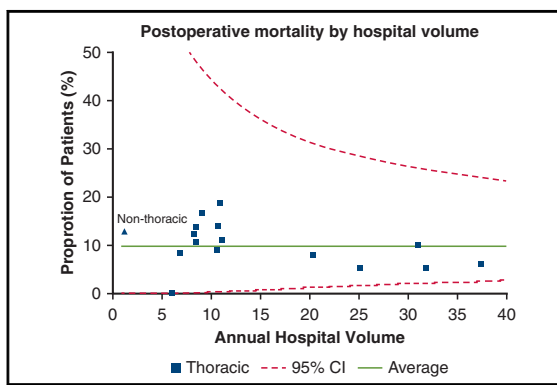

Postoperative mortality by hospital volume at designated thoracic centers in Ontario. ${ }^{14}$

\section{CENTRAL MESSAGE \\ Regionalization may optimize patient outcomes by allowing a critical mass of specialty pro- viders to work together and gain expertise. Increasing volume alone does not guarantee optimal outcomes.}

This Invited Expert Opinion provides a perspective on the following paper: Circulation. 2019 Oct 8;14O(15):1239-1250 CIRCULATIONAHA.118.038867.

See Commentaries on pages 330 and 331.

consequences and economic implications for key stakeholders before deciding whether regionalization can be applied more broadly in the United States.

\section{Jules Lin, MD}

The goal of regionalization is to provide optimal patient care for those who require specialty services such as thoracic surgery. The ability of a surgeon and an institution to provide high-quality care and optimize patient outcomes is facilitated by having a sufficient volume of patients to allow the team to build expertise and the institution to provide necessary resources. A high-quality program attracts individuals with focused interest and expertise, which in turn increases the quality of care. Providing high-quality care requires a team of specialist providers, including surgeons, anesthesiologists, nurses, pathologists, radiologists, 
physiotherapists, as well as other allied health providers and the infrastructure and equipment required by these providers. A high-quality program is not just a high-volume program.

In 2005, the Program in Evidenced-Based Care together with the Surgical Oncology Program of Cancer Care Ontario published a document entitled "Thoracic Surgical Oncology Standards: Guideline Development and External Review-Methods and Results." In 2015, the document was reviewed, the literature was updated, but no significant changes were made. ${ }^{1}$ The question to be addressed was: "What is the optimum organization for the delivery of cancer-related thoracic surgery in Ontario?" The document consisted of a systematic review of the literature, synthesis of the evidence, and recommendations. After review by both Cancer Care Ontario and external stakeholders, the recommendations were endorsed and regionalization of thoracic surgical services was undertaken. It is important to note that in Canada, cardiac and general thoracic surgery are separate. Thoracic surgeons do not practice cardiac surgery and only rarely will a cardiac surgeon perform pulmonary surgery.

Cardiac surgery has been regionalized almost since its inception.

The thoracic surgery standards included statements about hospital volume but also about surgeon criteria (training and certification), hospital resources (operating room, endoscopy, bronchoscopy, dedicated inpatient ward), dedicated thoracic nurses, physiotherapists, anesthesiologists, pathologists, multidisciplinary intensive care, multidisciplinary cancer care, pulmonologists, gastroenterologists, diagnostic and interventional radiology, and a formal relationship with a regional cancer center. The Standards related more to the "package" and "the team," whereas volume requirements were only one small component of the document.

Why was this work undertaken? There had been several reports in the literature suggesting the patient outcomes were improved if they received their surgery in highvolume hospitals. With respect to cancer surgery, Begg and colleagues ${ }^{2}$ reported a $17.3 \%$ operative mortality for esophagectomy performed at low-volume hospitals compared with $3.4 \%$ in high-volume hospitals. The volume "cut-off" varied by procedure but for esophagectomy the cutoff appeared to be $\geq 6$. Birkmeyer and colleagues, ${ }^{3}$ reporting on 14 types of surgery, found that for complex cases, in particular esophagectomy and pneumonectomy, hospitals that performed a greater number of those cases had improved patient outcomes. However, even more provocative was the 2003 report that surgeon volume accounted for a large proportion of the difference in mortality outcome between high- and low-volume hospitals (46\% for esophagectomy, $24 \%$ for lung resection). ${ }^{4}$ A more recent report also confirmed the importance of surgeon volume but also identified that there may be socioeconomic factors that lead to patients going to low-volume surgeons. Interestingly, these authors identified that even for wedge excision, in-hospital mortality was greatest for low-volume $(2.9 \%)$ compared with medium-volume $(2.0 \%)$ or high-volume $(1.0 \%)$ surgeons. ${ }^{5}$ There is a lot of press about hospital volume but less so about individual surgeon volume. Certainly, for complex procedures, surgeon experience matters, as shown in the paper by Birkmeyer and colleagues ${ }^{4}$ but, surprisingly, even for less-complex procedures this may be true. ${ }^{5}$ It is not just hours of practice, but focused practice. Expert surgeons not only have had lots of practice, they are constantly innovating and improving, adjusting their technique to improve their outcomes.

The Province of Ontario has a land mass of 416,000 squares miles and a population of 14.6 million. Prior to publication of the thoracic standards document, there were 46 hospitals in the Province of Ontario performing thoracic surgery; however, there were 4 universityaffiliated hospitals with very high volumes (Figure 1). The question was raised whether reorganization of thoracic cancer service delivery would improve patient outcomes. Based on the literature review performed by the Program in Evidenced-Based Care, the expert panel recommended reorganization of thoracic surgery cancer care into level 1 centers (14 hospitals), which were staffed with at least 3 Royal College-certified general thoracic surgeons, and had the required hospital resources recommended in the Standards document. Level 1 centers were chosen based on those centers meeting the Thoracic Standards before the implementation of the standards. However, there were 3 smaller centers wherein the patient volume could not support 3 full-time thoracic surgeons that were designated level 2 centers. These centers were permitted to continue thoracic surgery services because 1 was affiliated with a university and medical school, 1 had a regional trauma center as well as a cancer center, and 1 was geographically remote and was affiliated with a regional cancer center.

Implementation of the standards was completed in 2007. These changes were supported by financial incentives and subsequently financial penalties for noncompliance. ${ }^{6}$ Ontario has a single-payer health insurance program, and all hospital programs are funded by the government. For oncology, radiation facilities had been regionalized in the province since their inception whereas medical oncology became largely regionalized and hospital based at a later time. With implementation of the Standards, thoracic surgical oncology joined the other oncology disciplines. The effect of implementation of the Thoracic Standards document was that some patients would have to travel to a thoracic center for their cancer surgery but the government supplied travel grants to offset the cost. Because of geography, Canadians are used to traveling great distances, whether for medical care or for their child's hockey game! For some surgeons there were some changes: one moved to 


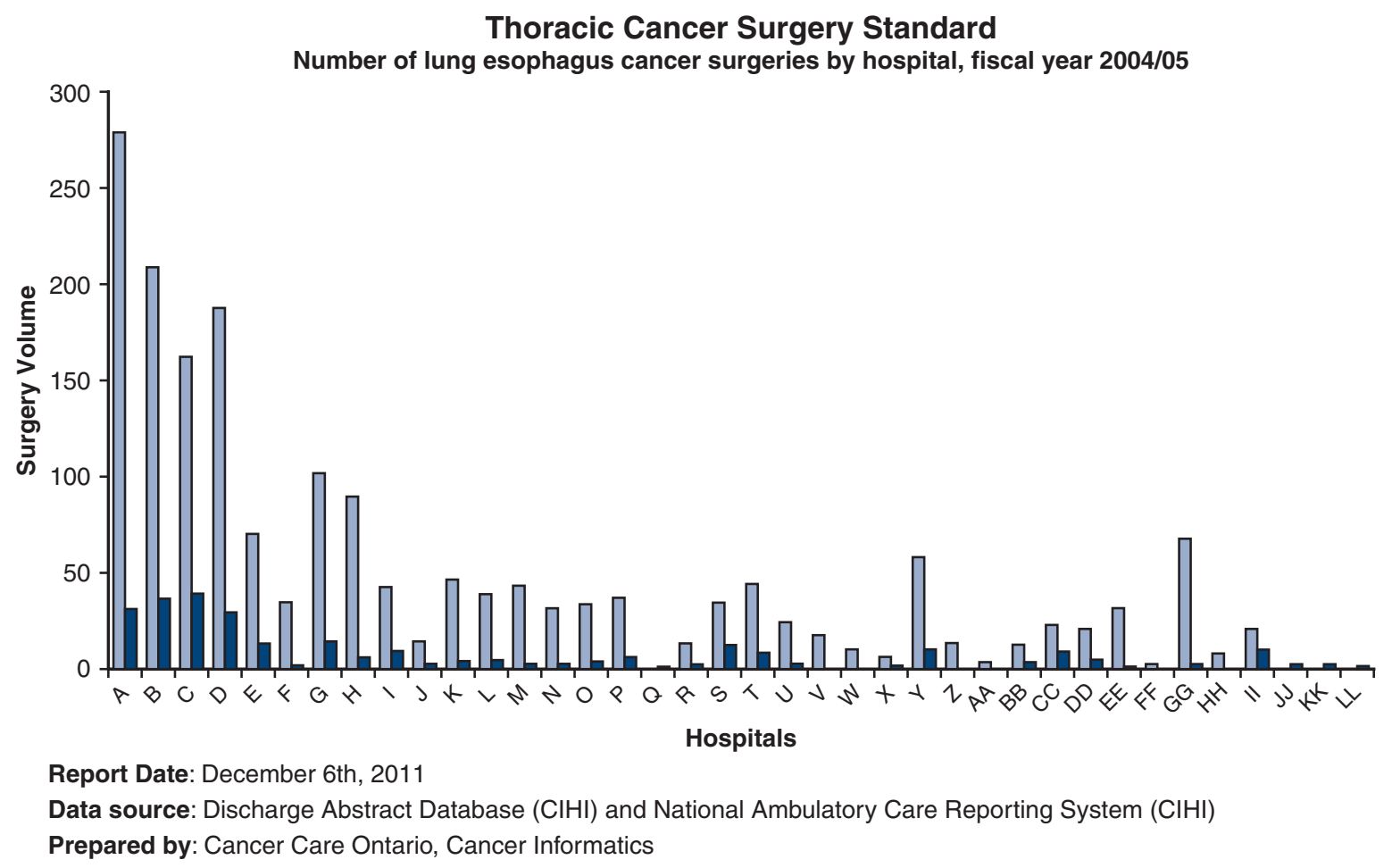

$\square$ Lung $\square$ Esophagus

FIGURE 1. Number of lung and esophageal cancer surgeries performed by hospital before regionalization.

a new center, whereas a few senior surgeons who had a combined general surgery and thoracic surgery practice stopped practicing thoracic surgery but continued general surgery. One surgeon continued to practice surgery at their original hospital but performed thoracic procedures at a nearby designated thoracic center.

Initially, there were some concerns about delivery of care in remote areas, particularly emergency care; however, the Ontario Ministry of Health had previously established a Critical Care Triage phone system (Criticall), wherein patients who are critically ill could be rapidly transported to other centers for care. This system facilitates emergent or urgent transfer of patients to institutions that can provide the care needed regardless of whether the patient is in a remote location or a smaller hospital without tertiary care services. A common argument against regionalization is that patient outcomes will be compromised by having to travel or be transferred; however, a recent paper evaluating this question in acute type $\mathrm{A}$ aortic dissection reported a $7.2 \%$ absolute reduction in operative mortality in the patients who were transferred to a high-volume center. ${ }^{7}$

In a Canada-wide study of pulmonary and esophageal resections, several papers have reported improved outcomes as a result of regionalization. ${ }^{8,9}$ For esophagectomy, high volume was defined as $>20$ esophagectomies per year (which was the volume requirement for level 1 centers in Ontario). The proportion of patients treated in a high- volume center increased from $29 \%$ to $61 \%$ between 1998 and 2007. An increase of 10 cases was associated with a $15 \%$ decrease in in-hospital mortality ( $95 \%$ confidence interval, $6 \%-23 \% P=.0010 .{ }^{8}$ The authors found that overall reduction in mortality was not related to increased volume in high-volume hospitals but rather referral of patients from low-volume centers to high-volume centers that already had good outcomes. This supports the concept of regionalization rather than just volume as a means of improving outcomes. ${ }^{8}$ Evaluation of the implementation of the Thoracic Standards in Ontario on outcomes of lung resection reported that trends to improve patient outcomes were already occurring before implementation of the Standards such that regionalization had no significant effect on mortality. ${ }^{10}$ Although not proven, it was suggested that since the majority of lung cancer surgery was already taking place at high-volume centers, changes due to regionalization were minimized, as the number of patients affected was actually quite small (Figure 2).

In other provinces in Canada, regionalization has taken place over time partly related to geography, and sparse population density, limited number of surgeons, and resource limitations. All provinces have now implemented some degree of regionalization: 4 centers in British Columbia, 2 in Alberta, 1 in each of Saskatchewan, Manitoba, Nova Scotia, and Newfoundland, while Quebec and New Brunswick are in transition. 


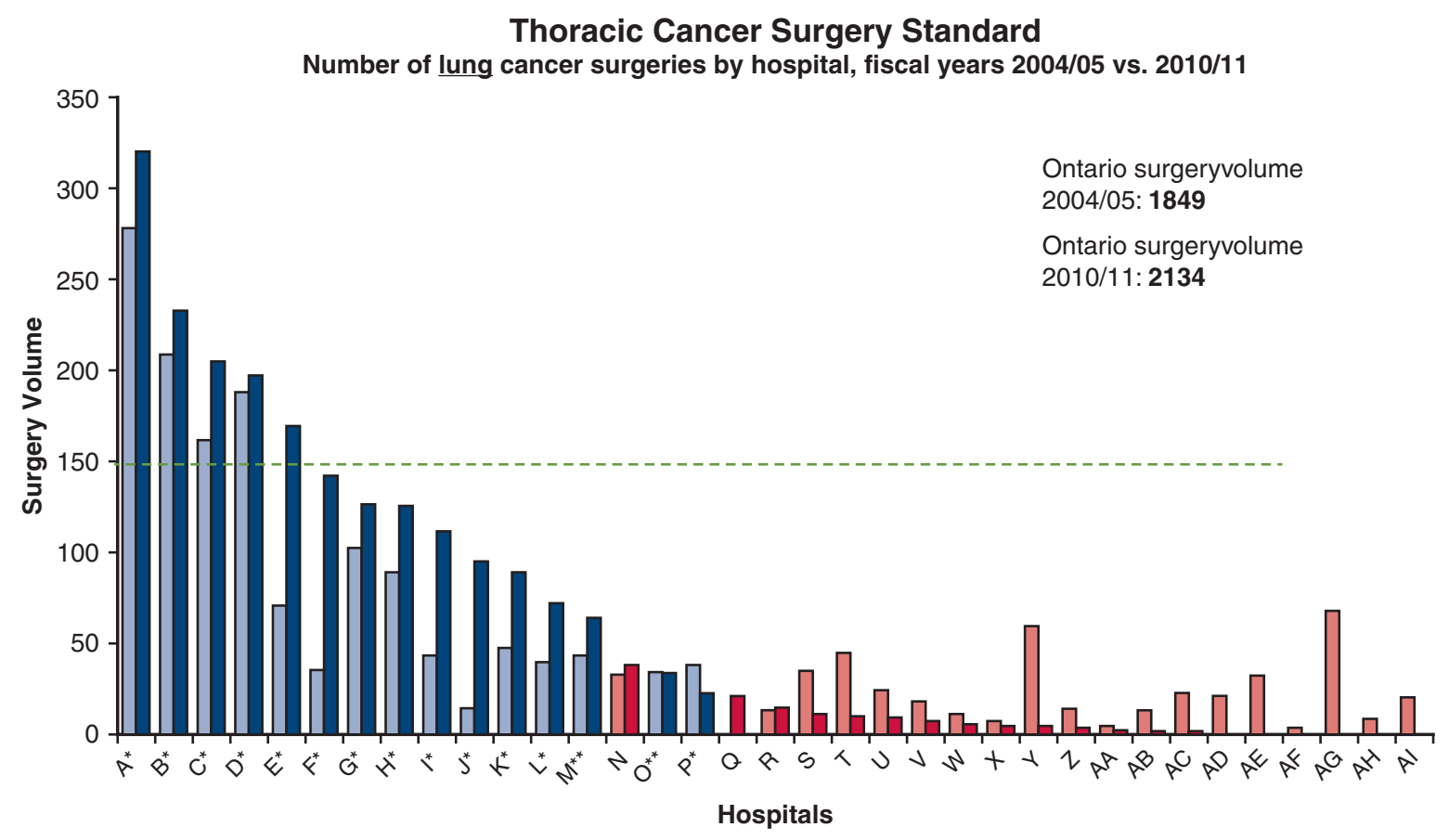

Report Date: December 6th, 2011

Data source: Discharge Abstract Database $(\mathrm{ClHI})$ and National Ambulatory Care Reporting System $(\mathrm{ClHI})$

Prepared by: Cancer Care Ontario, Cancer Informatics

Notes: 1 . *indicates level 1 designated centre $2 .{ }^{* *}$ indicates level 2 designated centre

$\square$ 04/05 Designated $\square 04 / 05$ Non-Designated
$\square$ 10/11 Designated $\quad \square$ 10/11 Non-Designated

FIGURE 2. Volume of lung cancer surgeries performed before and after regionalization.

In Ontario, esophagectomies are now performed only at designated centers; however, only 5 level 1 centers meet the target of 20 esophagectomies per year. These centers are the original 4 high-volume centers affiliated with universities and 1 additional center located in a region of high population growth (Figure 3). Other jurisdictions, including Ireland, the United Kingdom, and the Netherlands have also regionalized esophagectomy. The Association of Upper Gastrointestinal Surgeons of Great Britain and Ireland recommended that specialized units should have a combined gastric and esophageal resection volume of $>50$ cases per year. ${ }^{11}$ In the Republic of Ireland, there are now only 4 specialized upper gastrointestinal surgery units performing esophagectomy with significant improvements in outcome (Professor John Reynolds, oral communication, 2018). The Dutch Cancer Society proposed regionalization for complex cancer surgery and recommended a minimum of 20 esophagectomies per year. ${ }^{12}$ This has led to decreased mortality. ${ }^{13}$

Regionalization facilitates a critical mass of patients treated at a given center, which in turn leads to increased resources for the thoracic center in terms of hospital infrastructure and the attraction and promotion of specialty care, eg, thoracic anesthesia or pathology, interventional radiology, dedicated nursing, and intensive care. Creating thoracic centers promotes optimum cancer outcomes through multidisciplinary care and improves the ability to recognize and rescue patients when complications occur. However, a recent study has documented significant variation in esophagectomy practice among the level 1 centers, including the use of neoadjuvant therapy, lymph node harvest, positive margin rates, and in-hospital and 90-day mortality (unpublished results). ${ }^{14}$ This illustrates that volume does not necessarily confer quality or excellence (Figure 4). Much more work is needed to ensure the greatest quality of care, not just number of esophagectomies.

In Ontario, the level 1 centers were chosen based on already meeting the "thoracic standards" and these were the greater-volume hospitals in the province. All centers, including both level 1 and 2, were affiliated with a regional cancer center. Regional cancer centers provide radiation and medical oncology services. In most cases, a city has only one level 1 center but in the Greater Toronto area, which has a population of more than 3 million people, there are 3 level 1 centers within the Toronto city boundaries, whereas there are 5 level 1 centers outside the city in neighboring cities within a 1-hour drive. This reflects 
Thoracic Cancer Surgery Standard

Number of esophageal cancer surgeries by hospital, fiscal years 2004/05 vs. 2010/11

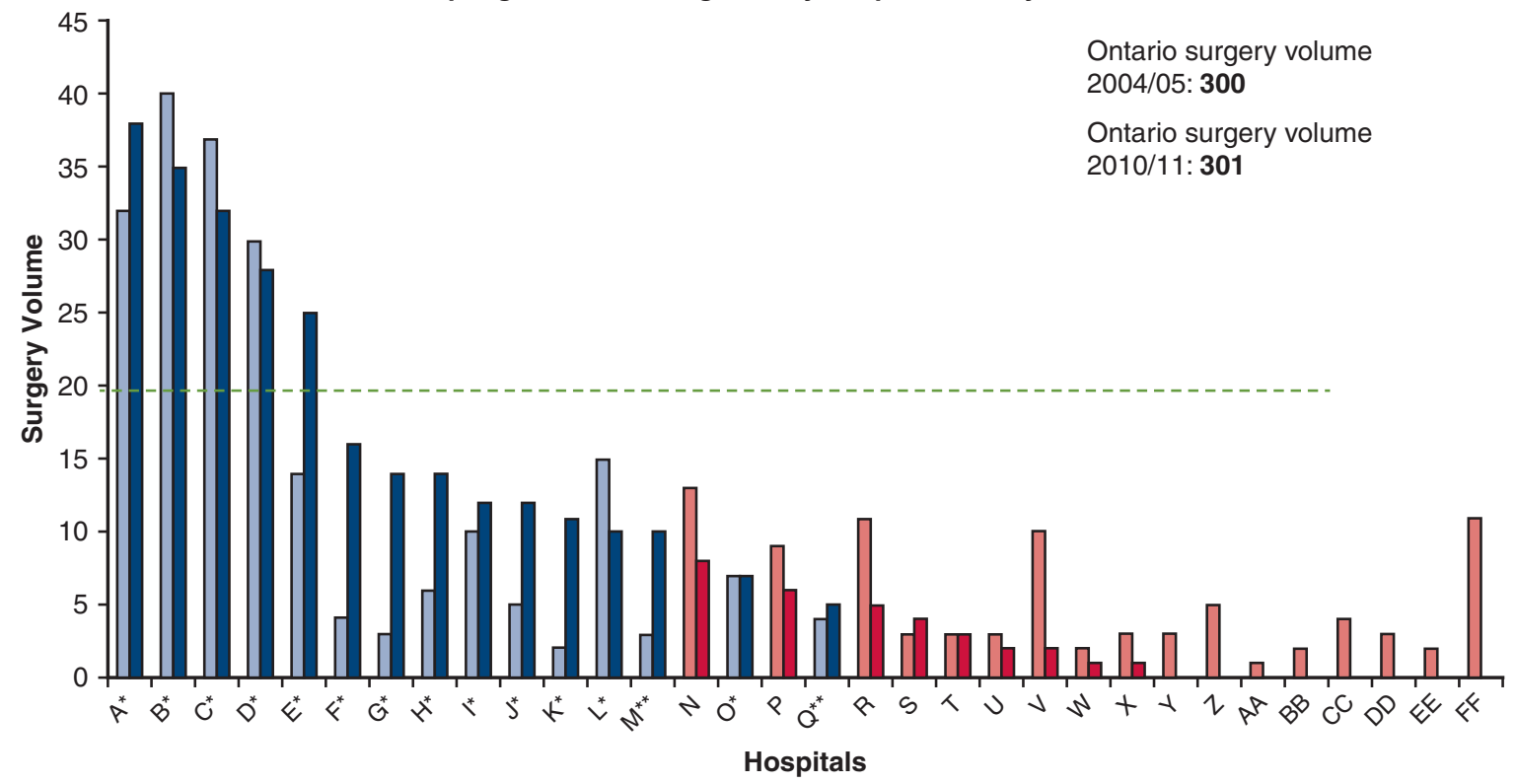

Report Date: December 6th, 2011

Data source: Discharge Abstract Database $(\mathrm{ClHI})$ and National Ambulatory Care Reporting System $(\mathrm{ClHI})$

Prepared by: Cancer Care Ontario, Cancer Informatics

Notes: 1. *indicates level 1 designated centre 2. **indicates level 2 designated centre

$\square$ 04/05 Designated $\square$ 04/05 Non-Designated
$\square$ 10/11 Designated $\square$ 10/11 Non-Designated

FIGURE 3. Number of esophagectomies performed at hospitals before and after regionalization.

the population density of the Greater Toronto Area and neighboring regions. It is assumed that all level 1 centers provide the same quality of care across the spectrum of thoracic diseases. There has been no attempt to have further specialization within centers, at least formally. Some centers have developed specialized areas of focus, for example,

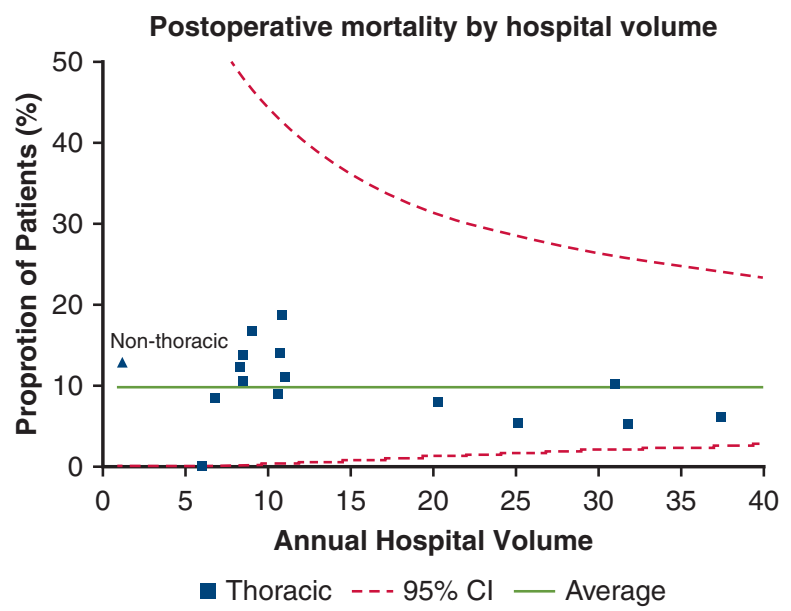

FIGURE 4. Postoperative mortality by hospital volume at designated thoracic centers in Ontario. lung transplantation is performed only at Toronto General Hospital, but this hospital still provides the breadth of thoracic surgical procedures.

From the patients' perspective, overall care is improved. Wait times for consultation and surgery have decreased through a process of organized evaluations and wait times administrative initiatives. Personally, I have not had anyone complain about having to travel to have their surgery performed by me. Patients are less happy with having to travel for tests or follow-up visits. This problem can easily be managed by arranging testing closer to home, using technical advances to "assess the patient" over "telehealth" or virtual visit applications. Potential arguments about fragmentation of care are overcome by involvement of local primary care providers, either physicians or nurse practitioners. However, the thoracic surgeon must be readily available to answer questions, provide advice, or take a patient back to the treating hospital when necessary. Suboptimal outcomes reported when patients are readmitted to their local hospital rather than the original surgical hospital can be avoided by open communication between surgeons and local providers as well as transfer of patients back to their original surgeon and treating hospital. In practice, patients are generally readmitted to 
the hospital where they had their original surgery or transferred to that hospital as soon as possible if they have been admitted elsewhere. Readmission rates have not increased. Nonthoracic centers are reluctant to care for thoracic patients, even for problems of a more general nature. Perhaps because of regionalization, they can refer the patient and so they chose to do so.

From the surgeons' perspective, our practice volumes are good and increasing overall. We have colleagues available to discuss or assist on difficult cases. We have colleagues who will provide care to our patients while we are at a conference or on a vacation. We are not sleep deprived from being up every night in the operating room on call. We work collaboratively with our thoracic surgical colleagues in the neighboring level 1 centers and have a formal relationship with a level 2 center such that complex patients can be transferred if necessary. The goal of regionalization was that $90 \%$ of thoracic oncology surgeries should be performed at designated centers, and this has largely been achieved (Figure 5). One hospital in the province continues to provide thoracic surgical services despite not being a designated center.

Can this model be applied to the American context? It is complicated. The United States has a large land mass but has a much greater population density compared with Canada. Thus, the geographic imperative does not exist for many of the states, although a few with smaller populations may be similar to the Canadian context. However, the concept of a critical mass of patients to support a highly skilled team with state-of-the-art resources applies to any complex health care problem. It is in this context that the Canadian model of regionalization can be applied. Optimizing patient outcomes is not just related to the number of procedures an individual surgeon performs or the number

\section{$\%$ of Thoracic Surgeries Performed in a} Designated Centre in Ontario

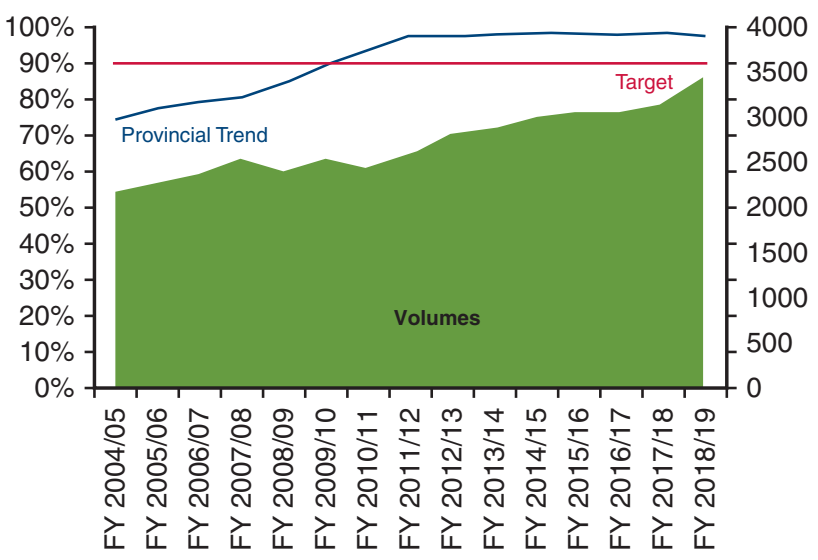

FIGURE 5. Percentage of thoracic cancer surgeries (lung and esophageal) performed in designated thoracic surgery centers by fiscal year May 2004 to January 2018. of procedures performed in the institution as a whole, but the complexity of those procedures, the complexity of the patients, and their comorbidities. Good outcomes depend on good judgment in terms of patient selection and choice of operation. Good judgment comes from experience-experience not just with straightforward simple procedures, but from complex challenging procedures. It is not just the experience of the surgeon that matters, but also the anesthesiologist and the anesthesia team who can handle any intraoperative situation. It is the skill and experience of the nursing team in the operating room, their familiarity with the type of procedure and equipment required. Nothing is more poetic than the symphony of coronary bypass surgery performed by an experienced team. This does not occur when a surgeon operates occasionally at a smaller hospital with a team who is not familiar with the surgeon, nor the surgeon with the team. I am sure we have all had the experience of operating in an institution other than our primary one, with staff you don't know, with equipment that is not what you are used to. Every step in the operation is just a little less smooth, less efficient, perhaps more stressful. After the procedure, it is the experience of the nursing team looking after the patient postoperatively who recognize when something isn't quite right and respond to a change in the patient's status. Nurses not familiar with the normal postoperative evolution of the patient will not recognize the subtle signs of trouble, which can then lead to delay in identification of a complication, leading to a greater likelihood of adverse outcome. It is the interventional radiology team who can reliably drain loculated collections or abscesses or biopsy small lung nodules without complications. It is the specialized lung pathologist who requests further testing on a lung specimen to determine epidermal growth factor receptor, Alk, or ROS 1 status, or programmed death-ligand 1 expression, who doesn't accept a diagnosis of poorly differentiated carcinoma but requests further immunohistochemical testing. It is the diagnostic radiologist who identifies that the pure ground-glass nodule now has a solid component or that the consolidated lower lobe has arterial supply from the aorta.

At a tertiary care center, we take all these skill sets for granted. As you read this, I am sure you are thinking, "of course, we have all that." We are all dedicated specialists, proud of what we do, confidant in our skills. But, we are human and thus imperfect. By having a specialized center with other experts around us, we have colleagues to help us when complications occur in the operating room or postoperatively. We have residents and fellows who ask questions and remind us of the relevant literature or clinical trial results. We have colleagues who are supportive but not afraid to point out where we could have done something differently. The real strength of regionalization is not specifically about volumes of surgery performed in an 
institution or by an individual surgeon but about critical mass and team expertise.

The single-payer system in Canada may facilitate regionalization. However, large health insurance companies and health care organizations in the United States have already started a process in some jurisdictions that may not be called regionalization but clearly reorganizes delivery of care. These organizations recognize that optimizing patient outcomes and economies of scale make sense from a financial perspective and will continue to drive reorganization of care. Complications and prolonged length of stay are costly. As already happens, the insurer will dictate where care occurs or which provider sees the patient. Optimizing patient outcomes benefits not just the patient but also the providers and insurers. Regionalization by whatever name, facilitates optimal delivery of complex surgical care by bringing together a critical mass of expertise across the entire thoracic team. Despite not having a single-payer system, this is already happening in the United States and should continue for the benefit of patients across the country.

\section{Conflict of Interest Statement}

The author reported no conflicts of interest.

The Journal policy requires editors and reviewers to disclose conflicts of interest and to decline handling or reviewing manuscripts for which they may have a conflict of interest. The editors and reviewers of this article have no conflicts of interest.

\section{References}

1. Cancer Care Ontario. Thoracic Surgical Oncology Standards. Available at: www. cancercareontario.ca/en/guidelines-advice/types-of-cancer/2171. Accessed January $16,2020$.
2. Begg CB, Cramer LD, Hoskins WJ, Brennan MF. Impact of hospital volume on operative mortality for major cancer surgery. JAMA. 1998;280:1747-51.

3. Birkmeyer JD, Siewers AE, Finlayson EV, Stukel TA, Lucas FL, Batista I, et al. Hospital volume and surgical mortality in the United States. N Engl J Med. 2002 346:1128-37.

4. Birkmeyer JD, Stukel TA, Siewers AE, Goodney PP, Wennberg DE, Lucas FL. Surgeon volume and operative mortality in the United States. $N$ Engl J Med. 2003;349:2117-23.

5. Harrison S, Sun T, Kamel M, Cleary C, Stiles BM, Altorki NK, et al. Do Individual surgeon volumes affect outcomes in thoracic surgery? Eur J Cardiothorac Surg. 2019;56:770-7

6. Sundaresan S, McLeod R, Irish J, Burns J, Hunter A, Meertens E, et al. Early results after regionalization of thoracic surgical practice in a single payer system. Ann Thor Surg. 2013;95:472-87; discussion 478-9.

7. Goldstone AB, Chui P, Balocchi M, Lingala B, Lee J, Rigdon J, et al. Interfacility transfer of care of Medicare beneficiaries with acute type A aortic dissection and regionalization of care in the United States. Circulation. 2019;140: 1239-50.

8. Finley CJ, Jacks L, Keshavjee S, Darling. The effect of regionalization on outcome in esophagectomy: a Canadian national study. Ann Thorac Surg. 2011;92:85-90; discussion 490 .

9. Finley CJ, Bendzsak A, Tomlinson G, et al. The effect of regionalization on outcome in pulmonary lobectomy: a Canadian National Study. J Thorac Cardiovasc Surg. 2010;140:757-63.

10. Bendzsak AM, Baxter NN, Darling GE, Keshavjee S, Urbach DR, Darling GE Regionalization and outcomes of lung cancer surgery in Ontario, Canada. J Clin Oncol. 2017;35:2772-80.

11. The Association of Upper Gastrointestinal Surgeons of Great Britain and Ireland The Provision of Services for Upper Gastrointestinal Surgery. Available at: https://www.augis.org/wp-content/uploads/2016/06/Provision-of-Services-June2016.pdf. Accessed January 16, 2020.

12. Busweiler LAD, Wijnhoven BPL, van Berge Henegouwen MI, Henneman D, Wouters MWJM, van Hilleergersurg R. The Dutch upper GI cancer audit: 2011-2014. J Clin Oncol. 2016;7(suppl):309.

13. Henneman D, Dikken JL, Putter H, Lemmens VEPP, Van der Geest LGM, van Hillegersberg R, et al. Centralization of esophagectomy: how far should we go? Ann Surg Oncol. 2014;21:4068-74.

14. Gupta V. Outcomes following resection for esophageal cancer in Ontario [dissertation]. Toronto, Canada: University of Toronto; 2020.

Key Words: regionalization, thoracic surgery, volume, thoracic standards 